\title{
Design and Analysis of Different Optical Attocells Deployment Models for Indoor Visible Light Communication System
}

\author{
M.S.M Gismalla ${ }^{1 *}$ M.F.L Abdullah' ${ }^{2}$, Mustafa Sami Ahmed², Wafi A Mabrouk², \\ Najib AL-Fadhali ${ }^{3}$, Elsadig Saeid ${ }^{4}$, A.S.M. Supa'at ${ }^{1}$, B Das $^{5}$
}

${ }^{1}$ School of Electrical Engineering, Faculty of Engineering,

Universiti Teknologi Malaysia, 81310 Skudai, MALAYSIA

${ }^{2}$ Department of Electronic Engineering, Faculty of Electrical and Electronic Engineering,

Universiti Tun Hussein Onn Malaysia (UTHM), Parit Raja, 86400 Batu Pahat, Johor, MALAYSIA

${ }^{3}$ Faculty of Engineering Technology,

Universiti Tun Hussein Onn Malaysia, Pagoh Education Hub, 84600 Pagoh, Muar, Johor, MALAYSIA

${ }^{4}$ Faculty of Engineering, Alzaeim Alazhari

University, Khartoum North, SUDAN

${ }^{5}$ Department of Electronic Engineering Quaid-e-Awam

University of Engineering, Science, and Technology Nawabhsh, Sindh, PAKISTAN

*Corresponding Author

DOI: https://doi.org/10.30880/ijie.2021.13.06.022

Received 14 September 2020; Accepted 27 June 2021; Available online 31 August 2021

\begin{abstract}
Visible light communication (VLC) is a promising candidate that is expected to revolutionize indoor environment communications performance and fulfill fifth generation and beyond (5GB) technologies requirements. It offers high and free bandwidth, electromagnetic interference immunity, low-cost front end and low power consumption. Also, VLC has dual functions that could be utilized in both illumination and communication concurrently. The number of optical attocells (OAs) and their deployment in the room represent the main issue that should be taken into consideration in designing an optimal VLC system. In this paper, we have introduced a new model of five OAs in the typical room. In addition to an investigation of various optical attocells (OAs) deployment models, in which a multi-variable evaluation was performed in terms of received power, illumination, SNR and RMS delay spread in order to determine the optimal OAs model. Also, various modulation schemes performances were investigated which included NRZ-OOK, BPSK, and QPSK in order to improve the BER performance. Results indicated that BPSK modulation had superior BER performance when compared with all OAs models. Further, a comprehensive results analysis and comparison of all proposed models was conducted over various parameters, in which our new proposed OAs model achieved an optimal performance in comparison with the other models.
\end{abstract}

\section{Introduction}

The ongoing exponential growth of data traffic, mobile devices, and wireless services produced a massive demand for new technologies to meet those demands and could support upcoming fifth generation and beyond (5GB). However, the current state of radio frequency (RF) technologies is suffering from bandwidth limitations, capacity crunches, high delays, and parasitic interferences [2]-[6]. Therefore, emerging, novel and revolutionary technologies such as visible light communication (VLC) have been proposed to complement RF technology's shortcomings within indoor short-range 
communication applications. VLC is a potential candidate for 5GB networks, and this is due to its valuable advantages and benefits that comprise of large and free bandwidth, cost-effectiveness, low power consumption, and electromagnetic interference immunity [6], [7]. Historically basic concepts and several applications of VLC technology were firstly introduced in [6], [8]-[10]. Concepts of infrared (IR) and VLC channel modeling were extensively reviewed for both indoor and vehicular applications. They were compared and investigated in terms of their advantages, drawbacks, similarities and differences [11], [12].

The optical attocell (OA) is defined as a light-emitting diodes (LEDs) array or LED bulb/lamb that can be placed on a room's ceiling to give illumination and communication simultaneously [13]-[15], and it shares many similarities with cellular network's base station (BS). Moreover, various studies were conducted that used many OAs deployments in a typical room model to improve the overall system performance in terms of illumination distribution, communication links, and overall performance quality, as introduced in [16]-[19].

\section{Related Work}

The number of OAs and their distribution in an indoor environment (i.e. room) play a significant role in VLC system design. Thus, various solutions have been implemented to improve the VLC system performance. One proposed solution implemented attocell minimization and maximization methods for accessing users in [20], in which four OAs were used. Alternatively, another model with four OAs that consists of different deployment scenarios was discussed in [21], [22], and the performance was analyzed in terms of received power and SNR. Further, in [23], different OAs configuration with various deployment scenarios was investigated to improve received power, coverage area, and power consumption, in which OAs positions have been optimized.

Also, the tradeoff between SNR, uniformity, and energy efficiency was investigated for four, nine, and sixteen OAs [24], [25], and semi-angle at half power were optimized to enhance the VLC system performance in the room. six OAs with three different scenarios were studied to optimize the received power and distribution of SNR [26]. Moreover, a multi-objective optimization approach was utilized in order to improve received power and SNR distributions with minimum power consumption [27]. Further, an inverse design of OAs deployment has been investigated to enhance the SNR and reduces the delay in [28].

On the other hand, various modulation techniques could be implemented to improve the performance of VLC communication system [29]. The OOK (On-Off-Keying) and BPSK (binary phase-shift keying) modulation techniques were investigated for the VLC system, both were evaluated in terms of voltage against distance in [30]. Results indicated that BPSK could be utilized in the VLC systems and it has the potential to outperform OOK in terms of far communication link ranges. Alternatively, spatial modulation technique was investigated for an indoor VLC system as well. It was simulated and evaluated based on its theoretical derived symbol error rate (SER) expression in [31]. In [32]-[34], additionally, the effect of different modulation techniques on data transmission, dimming, and bandwidth was investigated. Besides [35], in which a new approach was utilized to study the BER performance of the VLC system for LOS and Non-LOS links. Despite all previous investigations, there are no extensive results and discussions about determining the optimal number of OAs that could be deployed in a typical room model to deliver better illumination and communication performances. This paper is an expansion of original work presented in [18], [36], where different modulation techniques, as well as additional system parameters, are investigated to evaluate the VLC system performance in a typical room model. The main contributions of this paper comprise of a new OAs model design for the room ceiling, in addition to illumination and communication performances investigations of different OAs deployments for a typical room model's ceiling, with a comparison of the new proposed OAs design model results with contemporary models. Also, we have investigated the influence of semi-angle at half power on the behavior of illumination, SNR, and received power distribution, in addition to BER at different modulation schemes.

The remainder of this paper is organized as follows; Section 2 illustrates the related works. Section 3 introduces the system model, which includes received power, SNR, Modulation techniques, and RMS delay calculation. Followed by section 4 , in which results and analysis of different models are presented. Lastly, the paper is concluded and summarized in section 5 .

\section{System Model}

Fig. 1 illustrates fundamental VLC system constituents in a typical room setting with OAs installed on the room's ceiling, where the user or device will be at any position in the receiver plane within the room. The room model size is typically set as $5 \times 5 \times 3 \mathrm{~m}$, and it represents the room's length, width, and height, respectively. The transmitted signals will reach the receiver end by using a direct link which is termed the line of sight (LOS) link or reflected link and its termed as non-line of sight (Non-LOS) link [35]. We have considered the LOS link in the evaluation of this paper.

The deployment of OAs in the room's ceiling is illustrated in Fig. 2, where four, five, nine, and sixteen OAs models are employed, as shown in (a), (b), (c), and (d) respectively. The results of different OAs in the room have been discussed, analyzed, and compared in terms of SNR, received power, and BER performances. 


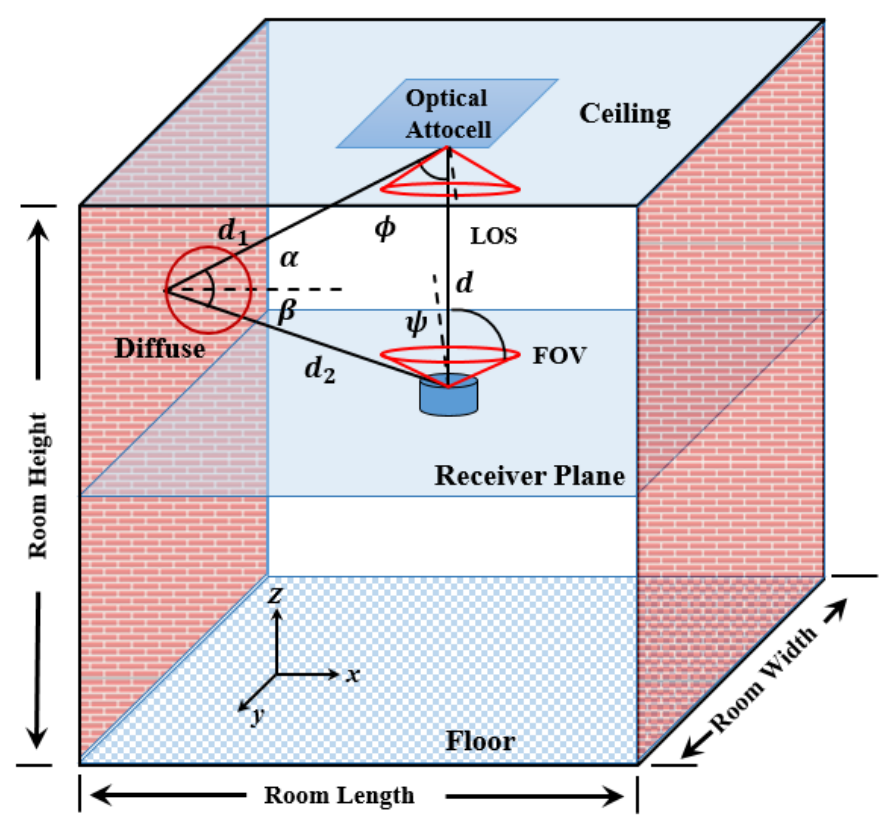

Fig. 1 - VLC system model.

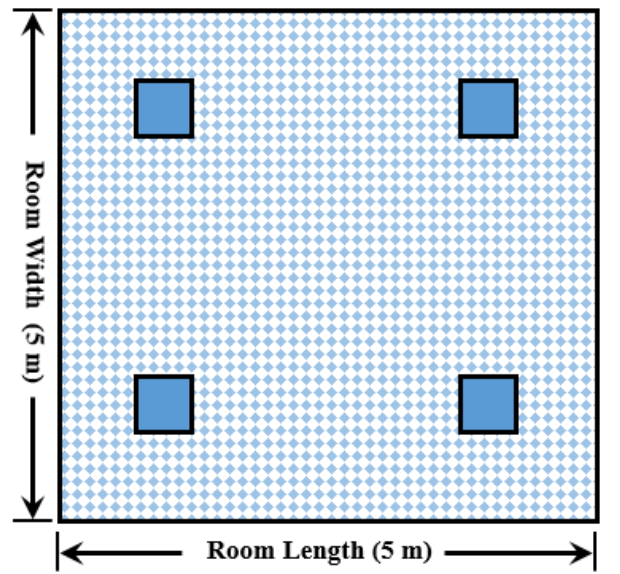

(a)

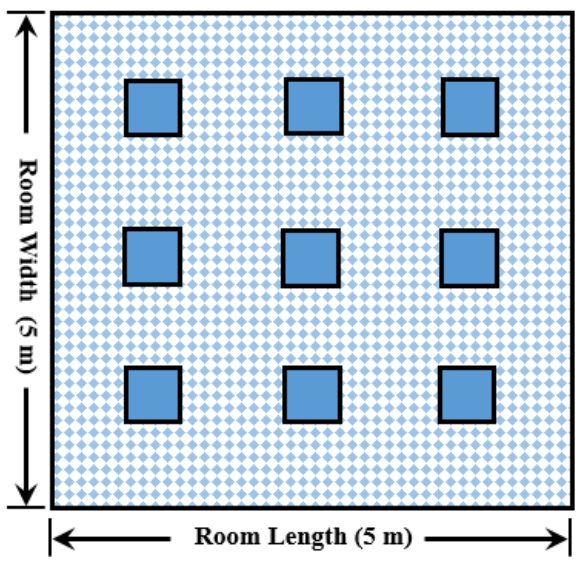

(c)

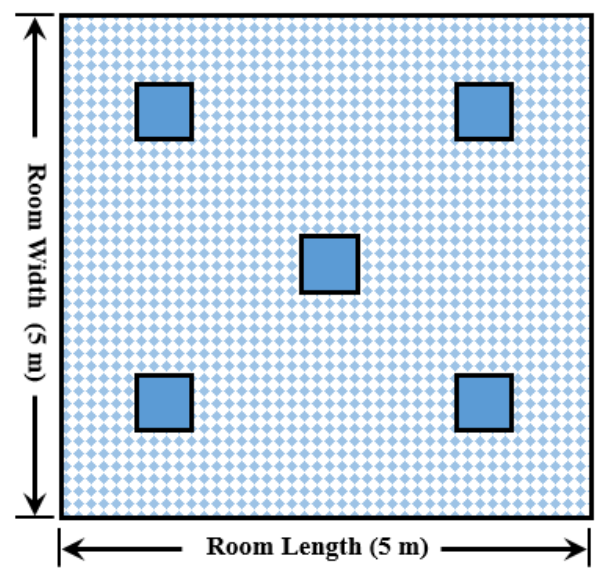

(b)

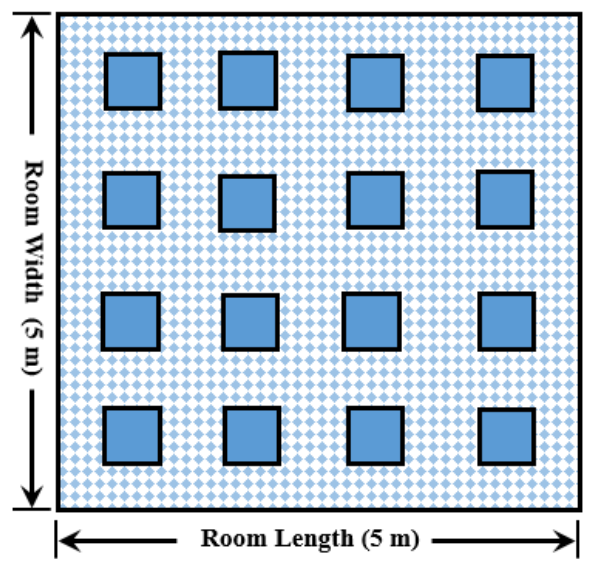

(d)

Fig. 2 - Deployment of OAs on the ceiling of the room (a) four OAs; (b) proposed model (five) OAs [17]; (c) nine OAs; (d) sixteen OAs [18] 
The new proposed model consists of five OAs deployed on the room's ceiling at a height of $3 \mathrm{~m}$ from the floor and $2.15 \mathrm{~m}$ from the receiver plane. Each OA has 2500 LED chips $(50 \times 50)$, and the distance between each cell is $1 \mathrm{~cm}$. The transmitted power per LED chip is $20 \mathrm{mw}$, and the center luminous intensity is $0.73 \mathrm{~cd}$. All these parameters are listed in Table 1.

Table 1 - Simulation parameters I

\begin{tabular}{l|l}
\hline Parameters & Values \\
\hline Room dimension & $5 \times 5 \times 3 m$ \\
Transmitted power per LED chip & $20[\mathrm{~mW}]$ \\
Number of LED chips per OA & $2500(50 \times 50)$ \\
Semi-angle at half power & $70[$ Degree $]$ \\
Size of OA & $0.49 \times 0.49$ \\
Central luminous intensity & $0.73[\mathrm{~cd}]$ \\
Height of receiver level & $0.85[\mathrm{~m}]$ \\
\hline
\end{tabular}

The Lambertian emission pattern is assumed as a source of radiation, and the radiation intensity of illuminance at the receiving plane is computed according to [37] as :

$$
I(\phi)=I(0) \cos ^{m}(\phi)
$$

where $\phi$ and $I(0)$ are the irradiance angle and center luminous intensity respectively; $m$ represents the Lambertian radiant order, and $\phi_{\frac{1}{2}}$ is LEDs semi-angle at half illumination as shown in Equation (2):

$$
m=\frac{-\ln 2}{\ln \left(\cos \phi_{\frac{1}{2}}\right)}
$$

Thus, horizontal illuminance level ( $E_{\text {hor }}$ ) for LOS link can be computed as expressed in (3) as

$$
E_{\text {hor }}=\frac{I(0) \cos ^{m}(\phi)}{D_{d^{2}}^{2}} \cdot \cos (\psi)
$$

where $\phi, I(0)$, and $\mathrm{m}$ are defined in Equation (1) and $D_{d}$ is the direct distance between OA and receiver.

Fig. 3 illustrates the behavior of average illuminance versus several semi-angle at half power in the entire room for the four models. According to the International Organization for Standardization (ISO), the required illuminance level for a typical room model of $(5 \times 5 \times 3 \mathrm{~m})$ ranges between 300 to 1500 Lux. The average illuminance of 702.89, 635.61, 750.09, and 863.65 Lux were obtained for four, five, nine, and sixteen OAs respectively. Therefore, the illuminance result of the four models was satisfied with the required illuminance based on ISO and could be used in a typical room

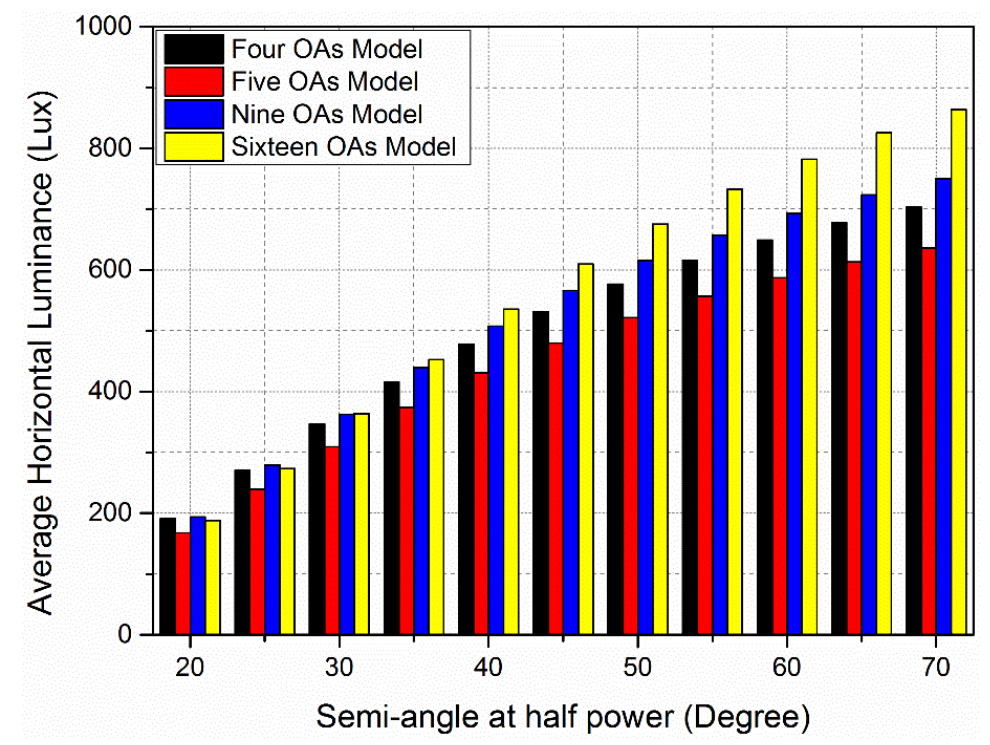

Fig. 3 - Average illumination distribution of all models in the typical room 


\subsection{Received Power and SNR Calculations}

To evaluate the quality of the VLC communication link, received power and SNR were measured at the receiving side. The received power $\left(P_{r}\right)$ was computed by utilizing the following expression:

$$
P_{r}=H(0) * P_{t}
$$

Where $P_{t}$ represents the transmitted power of OA; $H(0)$ is the channel DC gain for LOS link and it is given by:

$$
H(0)=\left\{\begin{array}{lr}
\frac{(m+1) A}{2 \pi D_{d}{ }^{2}} \cos ^{m}(\varnothing) T_{s}(\psi) g(\psi) \cos (\psi), 0 \leq \psi \leq \phi_{\frac{1}{2}} \\
0, & \psi>\phi_{\frac{1}{2}}
\end{array}\right.
$$

$D_{d}$ is the direct distance from OA to the receiver; whereas A denotes the physical area of the detector at the receiving side. The definition of all other parameters was presented in [17], [18]. Moreover, SNR at the receiver side is calculated at the presence of shot and thermal noises, and it is given by:

$$
S N R=\frac{\left[R P_{r}\right]^{2}}{\sigma_{\text {shot }}^{2}+\sigma_{\text {thermal }}^{2}}
$$

Where $\mathrm{R}$ is the responsivity of the receiver; $P_{r}$ is received power defined from Equation (4). The $\sigma_{\text {thermal }}^{2}$ is thermal noise variance; $\sigma_{\text {shot }}^{2}$ refers to shot noise variance; and all the parameters of the following equations were defined previously in [17], [18]. The variance of both noises is given as follows:

$$
\begin{gathered}
\sigma_{\text {shot }}^{2}=2 q\left[R P_{r}+I_{b g} I_{2}\right] B \\
\sigma_{\text {thermal }}^{2}=8 \pi k T_{k} \mathrm{\eta} A B^{2}\left(\frac{I_{2}}{G}+\frac{2 \pi \Gamma}{g_{m}} \mathrm{\eta} A I_{3} B\right)
\end{gathered}
$$

where $q$ is the electronic charge; $B$ refers to equivalent noise bandwidth; $I_{b g}$ and $I_{2}$ are the current of background light and bandwidth factor, respectively; $k$ is Boltzmann constant; $T_{k}, A$ and $\eta$ refer to absolute temperature; PD physical area, and fixed capacitance, respectively; $G$ and $g_{m}$ are the voltage gain and FET transconductance.

Parameters listed in Table 2 were used in our analysis. The physical detector area is set to $1 \mathrm{~cm}^{2}$, and the refractive index and optical concentrator gain are 1.5 and 1, respectively. Other relevant parameters used are concluded in Table 2 .

Table 2 - Simulation parameters II

\begin{tabular}{l|l}
\hline Parameters & \multicolumn{1}{|c}{ Values } \\
\hline Detector physical area & $1\left[\mathrm{~cm}^{2}\right]$ \\
Refractive index & 1.5 \\
optical concentrator gain & 1 \\
Reflection coefficient & 0.8 \\
Responsivity of photodiode & 0.54 \\
Bandwidth factor (I2) & 0.562 \\
Absolute temperature & $298[\mathrm{~K}]$ \\
FET transconductance & {$[30 \mathrm{mS}]$} \\
Bandwidth factor $(\mathrm{I} 3)$ & 0.0868 \\
FET channel noise factor & 1.5 \\
fixed capacitance & $112[\mathrm{pF} / \mathrm{cm} 2]$ \\
Data Rate & $30[\mathrm{Mb} / \mathrm{s}]$ \\
\hline
\end{tabular}

\subsection{Modulation Techniques}

In order to improve BER performance, various modulation techniques were implemented over the transmitted signal. In this paper, different modulations schemes were discussed which include, non-return-to-zero OOK (NRZ-OOK), binary, and quadrature phase-shift keying (BPSK) and (QPSK) [38], respectively. Therefore, BER or the corresponding probability of error performance of all three modulation techniques can be calculated by the following expressions:

$$
B E R_{N R Z-O O K}=\frac{1}{2} \operatorname{erfc}\left(\frac{1}{2 \sqrt{2}} \sqrt{S N R}\right)
$$




$$
\begin{gathered}
B E R_{B P S K}=\frac{1}{2} \operatorname{erfc}(\sqrt{S N R}) \\
B E R_{Q P S K}=\operatorname{erfc}(\sqrt{S N R})=2 B E R_{B P S K}
\end{gathered}
$$

\subsection{RMS Delay Spread}

The (RMS) root means square of delay spread has been employed to investigate interferences of adjacent OAs effects on the channel. Thus, in receiving end, the overall received power can be computed as follow:

$$
P_{r T}=\sum_{i=1}^{M} P_{d, i}+\sum_{j=1}^{N} P_{r e f, j}
$$

Where $M$ refers to the number of OA's direct links that are mounted on the ceiling to the receiver; $N$ denotes reflected away links from the wall to the receiver end number; $P_{d, i}$ relates to ith direct link received power; $P_{r e f, j}$ is the resulting received power from $j$ th reflected links. Hence, RMS delay and excess delay's mean are given as follows [37]:

$$
\mu=\frac{\sum_{i=1}^{M} P_{d, i} t_{d, i}+\sum_{j=1}^{N} P_{r e f, j} t_{r e f, j}}{P_{r T}}
$$

Accordingly, the RMS delay spread can be computed by:

$$
D_{r m s}=\sqrt{\mu^{2}-(\mu)^{2}}
$$

Where

$$
\mu^{2}=\frac{\sum_{i=1}^{M} P_{d, i} t_{d, i}^{2}+\sum_{j=1}^{N} P_{r e f, j} t_{r e f, j}^{2}}{P_{r T}}
$$

\section{Results and Analyses}

Various parameters were investigated to evaluate each proposed model performance, in which all four models satisfied illumination requirements, as shown in Fig. 3.

Moreover, received power distribution at semi-angle at half power of $70^{\circ}$ is shown in Fig. 4 , where the average received power values of $0.9625,2.458,1.6405$, and $0.7227 \mathrm{dBm}$ were achieved for four, five, nine, and sixteen OAs, respectively. In our proposed five OAs model, a better average received power was achieved with fluctuations between -1.286 and $5.9668 \mathrm{dBm}$, which represents the highest maximum and minimum received power, and this is due to the use of optimized FOV that presented in [17]. Further, the average, maximum, and minimum received power of each model are summarized in Table 3.

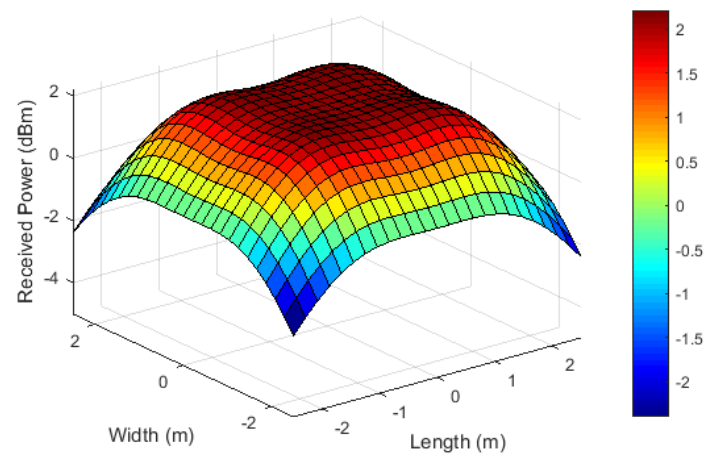

(a)

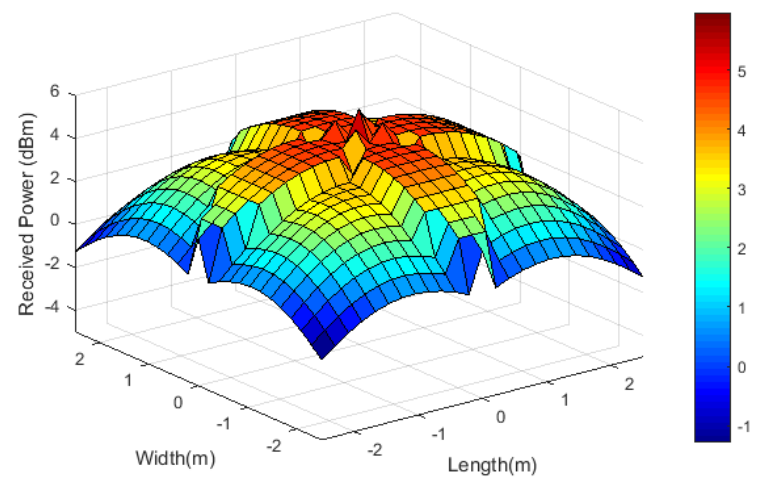

(b) 


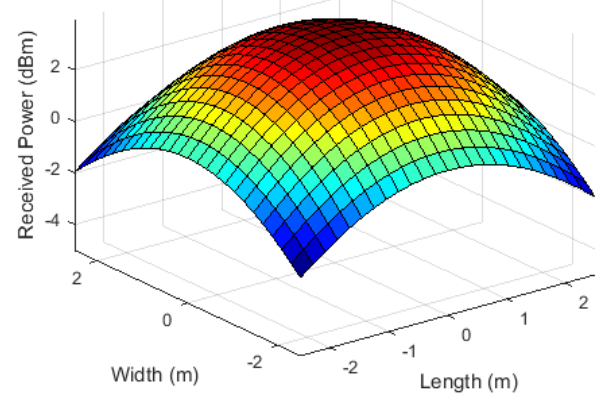

(c)
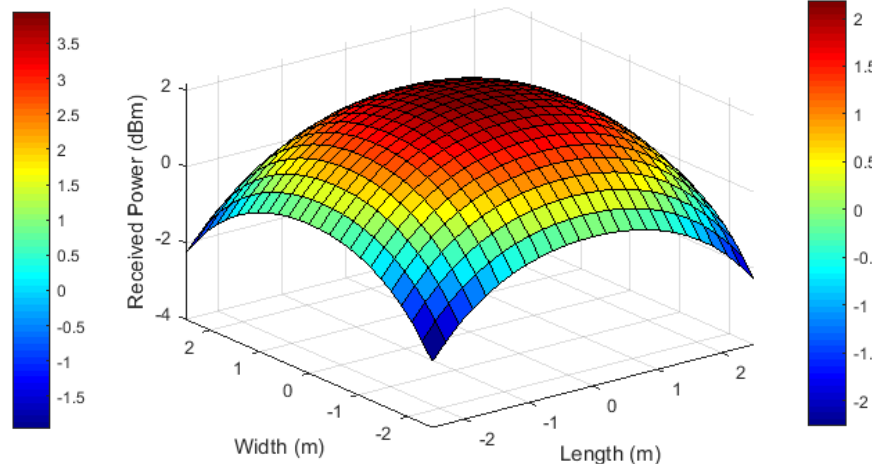

(d)

Fig. 4 - Received power distribution of (a) four, (b) five, (c) nine, and (d) sixteen OAs models.

Furthermore, Fig. 5 demonstrates the SNR distribution of all four models, in which average SNR values of 72.18, 74.58, 73.53, and 71.11 dB were achieved for four, five, nine, and sixteen OAs cases, respectively. Hence, five OAs have acquired the highest average, maximum, and minimum SNR compared to other models. Lastly, SNR values of all models are listed in Table 3.

Table 3 - Received power and SNR performances of the four models

\begin{tabular}{clcccc}
\hline Parameters & & Four OAs & Five OAs & Nine OAs & \multicolumn{2}{c}{ Sixteen OAs } \\
\hline \multirow{3}{*}{ Received power $(\mathrm{dBm})$} & Maximum & 2.209 & 5.9668 & 3.9491 & 2.1897 \\
\cline { 2 - 6 } & Minimum & -2.3986 & -1.286 & -1.9629 & -2.2683 \\
\cline { 2 - 6 } & Average & 0.9625 & 2.458 & 1.6405 & 0.7227 \\
\hline \multirow{2}{*}{ SNR $(\mathrm{dB})$} & Maximum & 74.6688 & 81.5945 & 78.1483 & 74.0418 \\
& Minimum & 65.4544 & 67.0911 & 66.3258 & 65.1267 \\
\cline { 2 - 6 } & Average & 72.1761 & 74.5782 & 73.5319 & 71.1081 \\
\hline
\end{tabular}

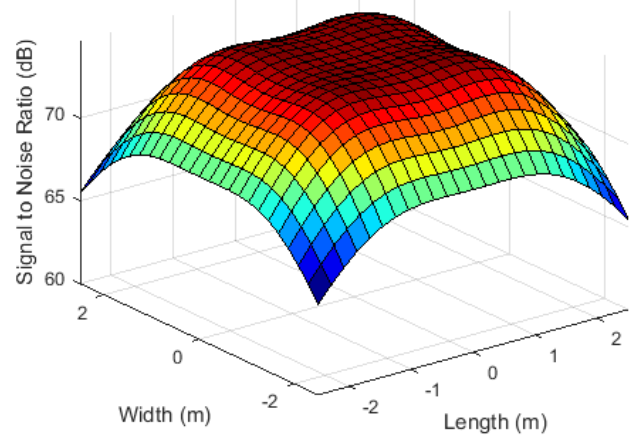

(a)

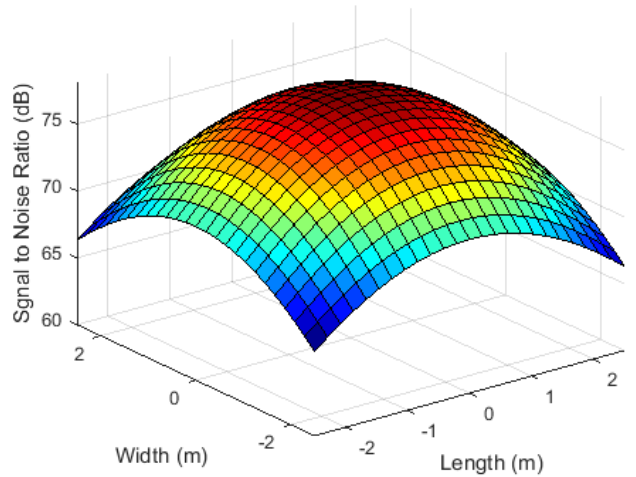

(c)

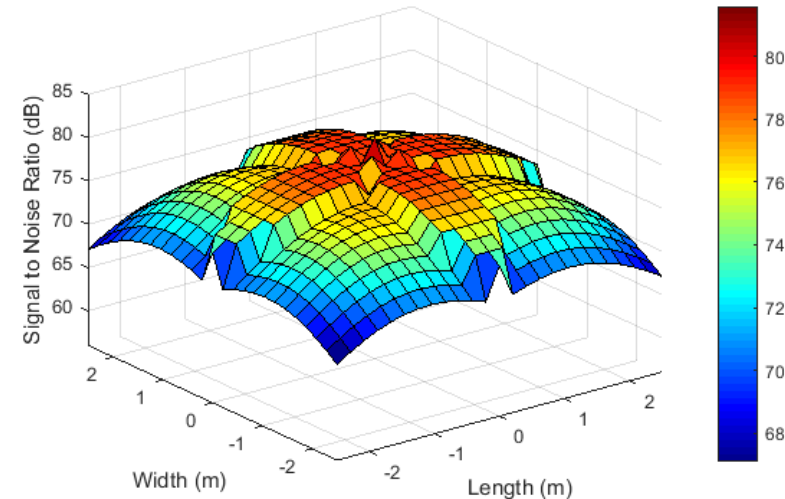

(b)
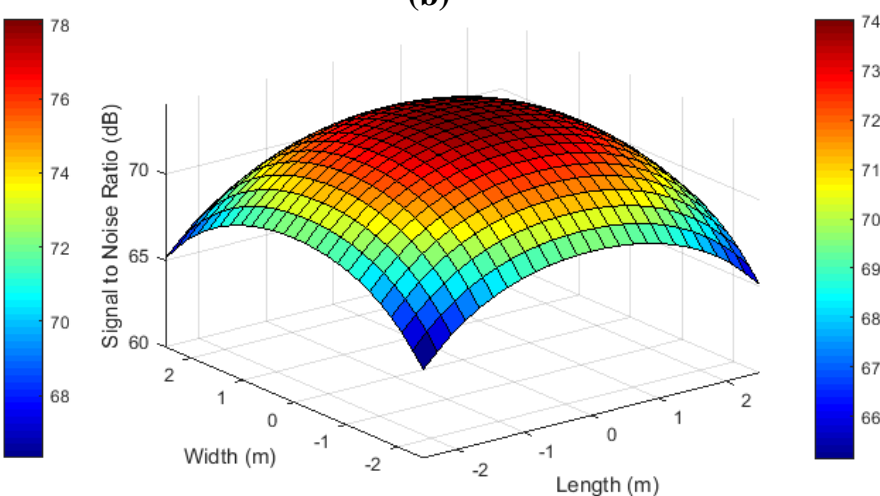

(d)

Fig. 5 - SNR distribution of (a) four; (b) five; (c) nine; (d) sixteen OAs models 
Fig. 6 shows the behavior of minimum SNR distribution and received power with respect to various semi-angle at half power in the entire room. Based on results, four, five, and nine OAs, small semi-angle at half power had the lowest received power and SNR levels, whereas received power increased as the semi-angle at half power increased. However, the sixteen OAs had better-received power compared to other models at small semi-angle at half power, and it slightly decreased as the semi-angle at half power increased. Results display that our proposed five OAs had better performance compared to other models, and the distribution of received power and SNR was fair for all models at semi-angle at half power of more than $40^{\circ}$, because for values beyond $40^{\circ}$ the whole room will be approximately covered and any increase doesn't significantly affect in the performance.

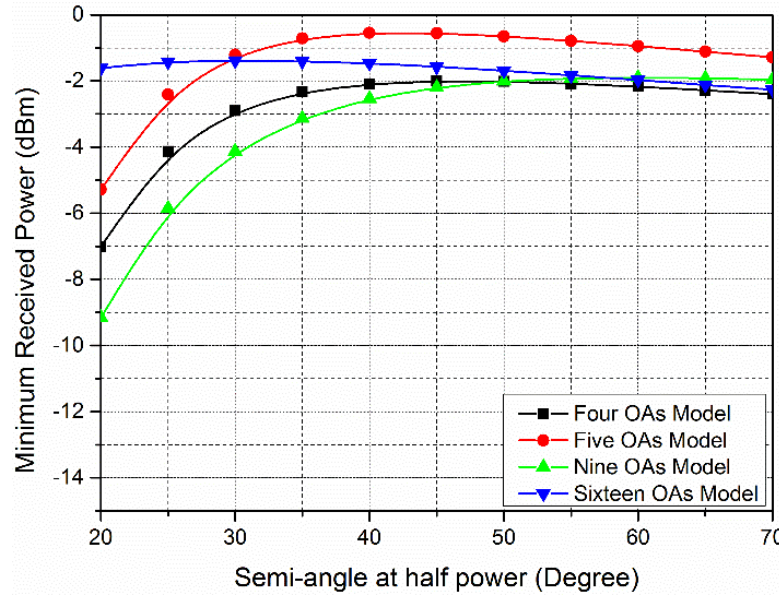

(a)

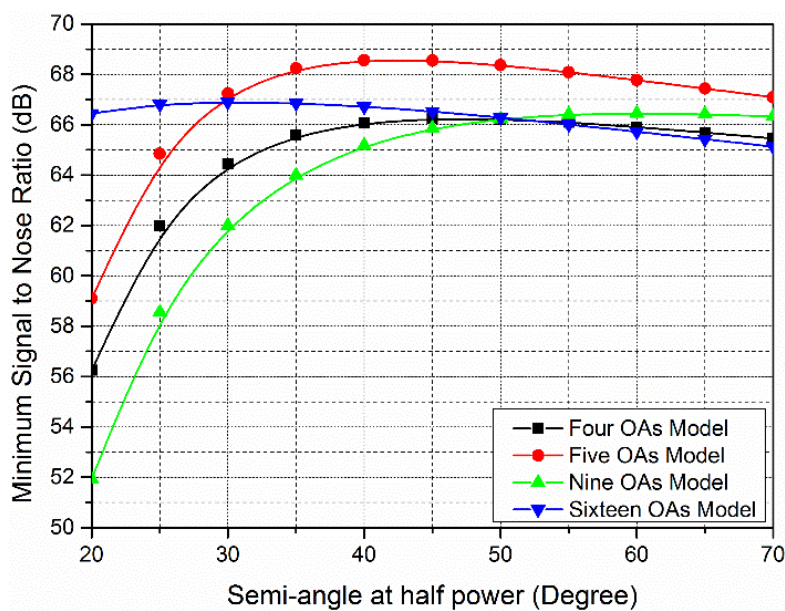

(b)

Fig. 6 - Distribution of minimum (a) received power; (b) SNR of all models versus various semi-angle at half power

Alternatively, in terms of BER performance models were also evaluated with various modulation techniques that employed NRZ-OOK, BPSK and QPSK schemes as shown in Fig. 7. Based on tabulated results in Table 4, measured BER levels of 5.16E-23, 8.57E-13, 1.63E-04, and 0.0092 were obtained utilizing BPSK modulation for four, five, nine, and sixteen OAs respectively, which had the best BER performance compared with other modulation schemes. Moreover, BER performance behavior under various modulations was also investigated, and results indicate that it improved with the increase of the semi-angle at half power increase, and it became slightly fair at angles above $40^{\circ}$. Therefore, based on BER performance, a lower number of OAs in the room needs more power compared to larger OAs numbers. However, it has significantly improved the BER and RMS delay due to its high transmitted power and safer distance between OAs. On the other hand, larger OAs distributions cause severe adjacent interference, which degrades performance and produces higher BER and RMS delay levels.

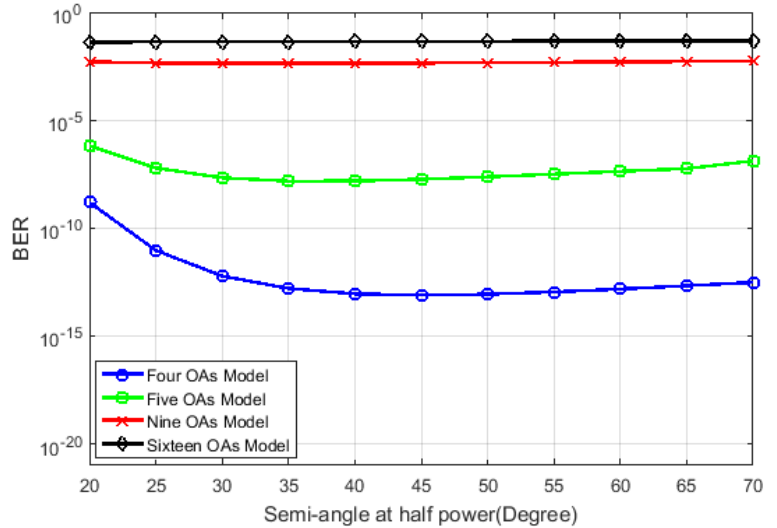

(a)

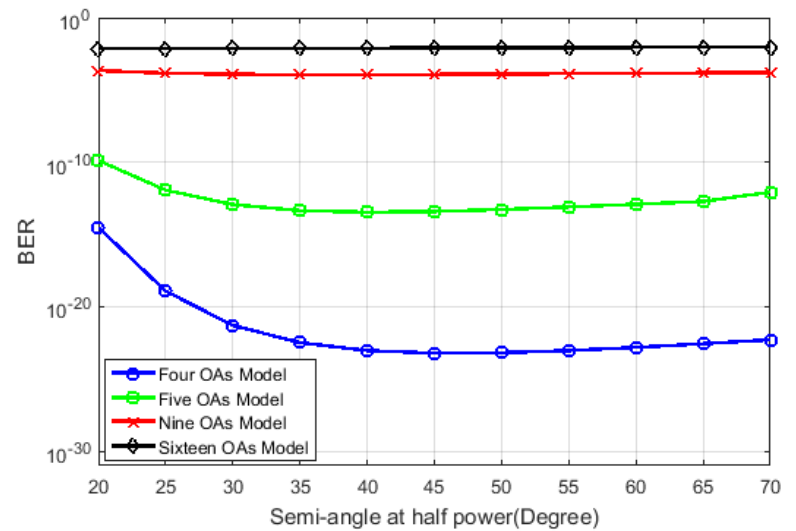

(b) 


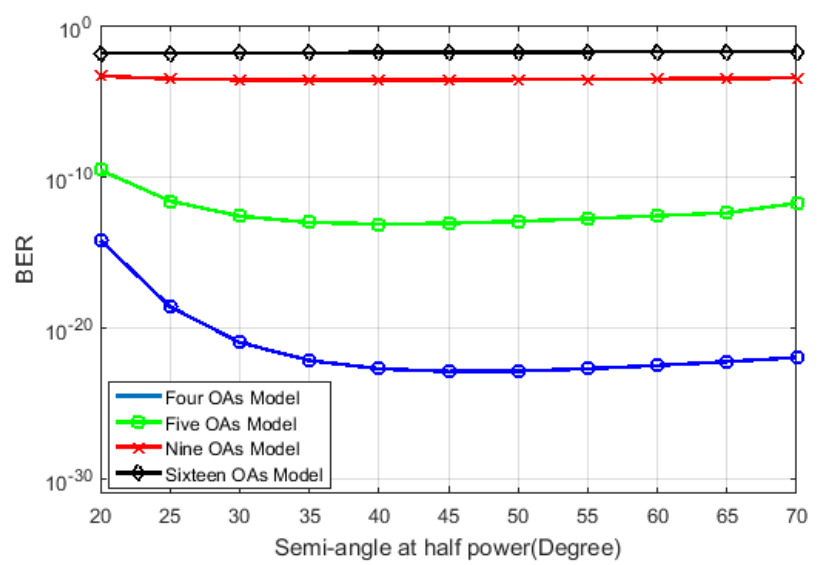

(c)

Fig. 7 - BER performance of (a) NRZ-OOK; (b) BPSK; (c) QPSK modulations versus various semi-angle at half power

The RMS delay spread of all four models is shown in Fig. 8 at a semi-angle at half power of $70^{\circ}$. Values of 1.0302 , $0.1375,0.5039$, and $1.2879 \mathrm{~ns}$ were achieved for four, five, nine, and sixteen OAs, respectively. It is noted that our new proposed model of five OAs had the lowest RMS delay compared with other models. Moreover, average RMS delay behavior under various semi-angle at half power is shown in Fig. 9, in which it increases with the increase in semi-angle at half power values. The deployment of a massive number of OAs in the room will cause severe interference that causes a delay on the receiving end. Thus, RMS delay could be decreased by choosing an appropriate number of OAs, to satisfy the required illuminance as well as establishing a reliable communication link. Moreover, five OAs models utilized 2500 LED chips per OA instead of 3600 LED chips per OA at the four OAs model, which results in OAs size reduction, therefore, produced the lowest interference or RMS delay over the existing four OAs model.

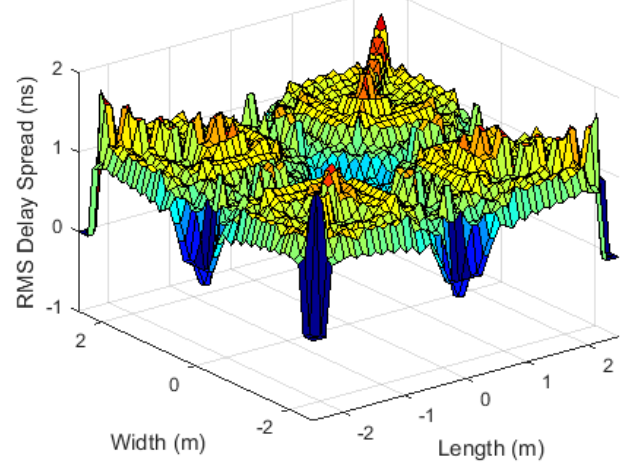

(a)

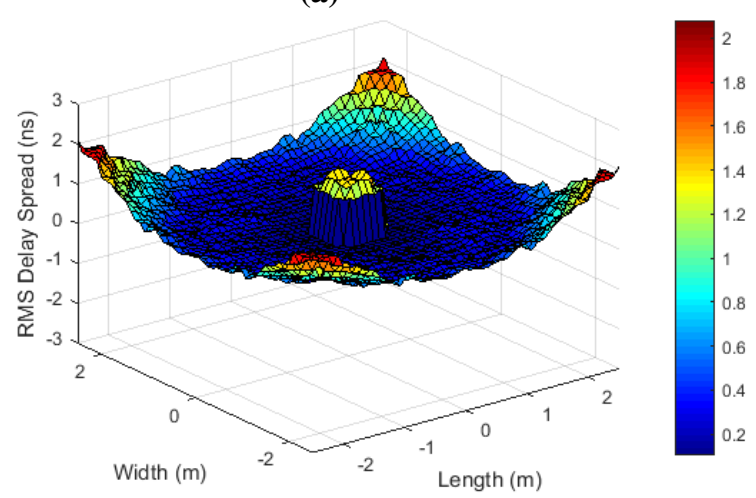

(c)

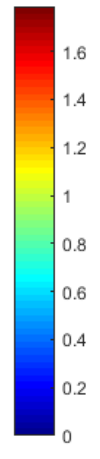

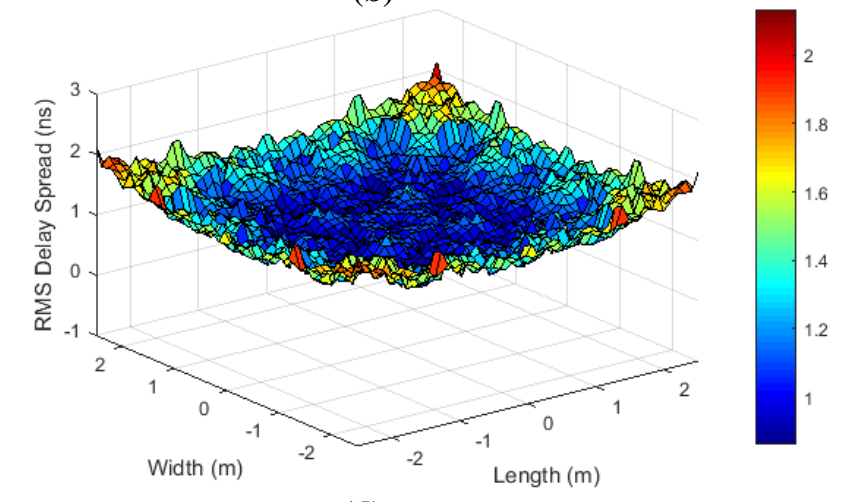

(d)

Fig. 8 - RMS delay spread distribution for (a) four; (b) five; (c) nine; (d) sixteen OAs models 


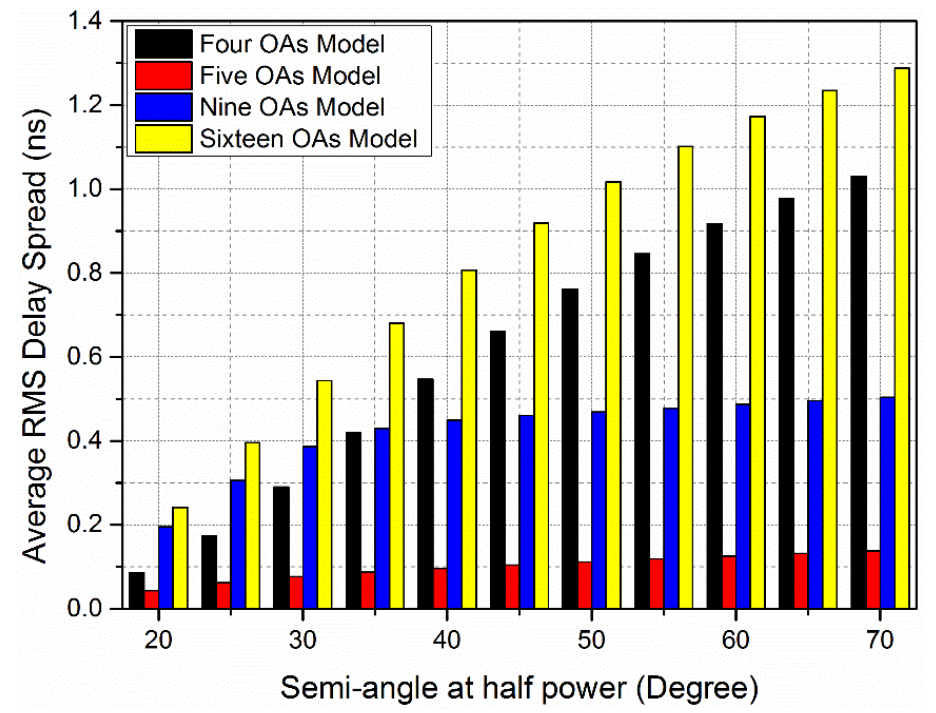

Fig. 9 - Average RMS delay spread versus different semi-angle at half power

BER performance for different modulation techniques, as well as RMS delay spread, are summarized in Table 4. BPSK shows a better BER performance over NRZ-OOK and QPSK schemes. Besides, five OAs achieved an overall better performance in terms of all evaluated parameters over other OAs models. Therefore, it could be employed in a typical room model to acquire optimum performance.

Table 4 - Performance of BER and RMS delay spread for the four models

\begin{tabular}{lllllc}
\hline & & Four OAs & Five OAs & Nine OAs & Sixteen OAs \\
\hline \multirow{3}{*}{ BER } & NRZ-OOK & $3.06 \mathrm{E}-13$ & $1.29 \mathrm{E}-07$ & 0.0054 & 0.0477 \\
\cline { 2 - 6 } & BPSK & $5.16 \mathrm{E}-23$ & $8.57 \mathrm{E}-13$ & $1.63 \mathrm{E}-04$ & 0.0092 \\
\cline { 2 - 6 } & QPSK & $1.03 \mathrm{E}-22$ & $1.71 \mathrm{E}-12$ & $3.26 \mathrm{E}-04$ & 0.0184 \\
\hline \multicolumn{2}{l}{ RMS delay spread $(\mathrm{ns})$} & 1.0302 & 0.1375 & 1.2879 & 0.5039 \\
\hline
\end{tabular}

\section{Conclusion}

This paper presents an investigation of different OAs deployment models in a typical room, with the intent to find the optimal number of OAs that could be employed for optimum illumination and communication performances. Thus, various contemporary VLC system models that consist of four, nine, and sixteen OAs were studied with respect to several previously discussed parameters. In this paper, we have evaluated the new model of five OAs and analyzed its results in addition to a comprehensive comparison with the previous models in terms of received power, illumination, SNR, and RMS delay spread. Besides, the employment of different modulation techniques that included NRZ-OOK, BPSK, and QPSK to acquire an improved BER performance. Best results of 5.16E-23, 8.57E-13, 1.63E-04, and 0.0092 BER levels were obtained when BPSK was utilized for four, five, nine, and sixteen OAs models, respectively. Thereby obtaining better BER performance for four and five OAs models that fulfill reliable communication link requirements of a BER value that is less than $10^{-6}$. Based on the previous investigations of new and previous OAs models, it is noted that it is impossible to achieve an overall optimal system performance by optimizing all relevant parameters. This is due to the pre-existing tradeoffs between some parameters (i.e. OAs numbers vs RMS delay) which makes it difficult to improve an aspect without degrading the others. Therefore, based on all models results comparisons, our new model of five OAs has produced a better performance for all studied parameters with an acceptable illumination performance. Future research will consider advanced modulation techniques and power consumption reduction mechanisms as means for further performance improvements.

\section{Acknowledgment}

The authors of this paper wish to acknowledge the funding of this project by the Research Management Centre (RMC) Universiti Teknologi Malaysia (UTM), Malaysia. Also, they wish to thank the Universiti Tun Hussein Onn Malaysia (UTHM) for their technical support of this project. 


\section{References}

[1] A. Al-Kinani, C.-X. Wang, L. Zhou, and W. Zhang, "Optical Wireless Communication Channel Measurements and Models," IEEE Commun. Surv. Tutorials, vol. 20, no. 3, pp. 1939-1962, 2018

[2] L. U. Khan, "Visible light communication: Applications, architecture, standardization and research challenges," Digit. Commun. Networks, vol. 3, no. 2, pp. 78-88, 2017

[3] S. Kumar and P. Singh, "A Comprehensive Survey of Visible Light Communication: Potential and Challenges," Wirel. Pers. Commun., no. 123456789, 2019

[4] J. J. Bao, Q. Mai, and J. F. Tu, "Study of cooperative strategy based on space-time labeling diversity in indoor visible light communication systems," Symmetry (Basel)., vol. 12, no. 5, 2020

[5] P. H. Pathak, X. Feng, P. Hu, and P. Mohapatra, "Visible light communication, networking, and sensing: A survey, potential and challenges," IEEE Commun. Surv. tutorials, vol. 17, no. 4, pp. 2047-2077, 2015

[6] L. E. M. Matheus, A. B. Vieira, L. F. M. Vieira, M. A. M. Vieira, and O. Gnawali, "Visible Light Communication: Concepts, Applications and Challenges," IEEE Commun. Surv. Tutorials, vol. 21, no. 4, pp. 3204-3237, 2019

[7] J. Lian, Z. Vatansever, M. Noshad, and M. Brandt-Pearce, "Indoor visible light communications, networking, and applications," J. Phys. Photonics, vol. 1, no. 1, p. 12001, 2019

[8] H. Burchardt, N. Serafimovski, D. Tsonev, S. Videv, and H. Haas, "VLC: Beyond point-to-point communication," IEEE Commun. Mag., vol. 52, no. 7, pp. 98-105, 2014

[9] A. Jovicic, J. Li, and T. Richardson, "Visible light communication: Opportunities, challenges and the path to market," IEEE Commun. Mag., vol. 51, no. 12, pp. 26-32, 2013

[10] A. M. Căilean and M. Dimian, "Impact of IEEE 802.15.7 standard on visible light communications usage in automotive applications," IEEE Commun. Mag., vol. 55, no. 4, pp. 169-175, 2017

[11] F. Miramirkhani and M. Uysal, "Channel modelling for indoor visible light communications," Philos. Trans. R. Soc. A Math. Phys. Eng. Sci., vol. 378, no. 2169, p. 20190187, Apr. 2020

[12] M. Karbalayghareh, F. Miramirkhani, H. B. Eldeeb, R. C. Kizilirmak, S. M. Sait, and M. Uysal, "Channel Modelling and Performance Limits of Vehicular Visible Light Communication Systems," IEEE Trans. Veh. Technol., vol. 9545, no. c, pp. 1-1, 2020

[13] M. S. M. Gismalla and M. F. L. Abdullah, "Performance evaluation of optical attocells configuration in an indoor visible light communication," Indones. J. Electr. Eng. Comput. Sci., vol. 14, no. 2, p. 668, May 2019

[14] D. Tsonev, S. Videv, and H. Haas, "Light fidelity (Li-Fi): towards all-optical networking," in Broadband Access Communication Technologies VIII, 2014, vol. 9007, p. 900702

[15] H. Kazemi, M. Safari, and H. Haas, “A Wireless Optical Backhaul Solution for Optical Attocell Networks,” IEEE Trans. Wirel. Commun., vol. 18, no. 2, pp. 807-823, 2019

[16] M. S. M. Gismalla, M. F. L. Abdullah, M. I. Niass, B. Das, and W. A. Mabrouk, "Improve uniformity for an indoor visible light communication system," Int. J. Commun. Syst., vol. 33, no. 8, p. e4349, May 2020

[17] M. S. M. Gismalla, M. F. L. Abdullah, B. Das, W. A. Mabrouk, and N. E. Mahfouz, "Design of an optical attocells configuration for an indoor visible light communications system," AEU - Int. J. Electron. Commun., vol. 112 , 2019

[18] M. Khadr, A. Abd El Aziz, H. Fayed, and M. Aly, "Bandwidth and BER Improvement Employing a PreEqualization Circuit with White LED Arrays in a MISO VLC System," Appl. Sci., vol. 9, no. 5, p. 986, Mar. 2019

[19] N. E. Mahfouz, H. A. Fayed, A. Abd El Aziz, and M. H. Aly, "Improved light uniformity and SNR employing new LED distribution pattern for indoor applications in VLC system," Opt. Quantum Electron., vol. 50, no. 9, p. 350,2018

[20] A. M. Vegni and M. Biagi, “Optimal LED placement in indoor VLC networks,” Opt. Express, vol. 27, no. 6, p. 8504, 2019

[21] T. Ganjian, G. Baghersalimi, and Z. Ghassemlooy, "Performance evaluation of the received power based on the transmitter position in a visible light communications system," in 2017 Iranian Conference on Electrical Engineering (ICEE), 2017, pp. 1763-1768

[22] F. Madani, G. Baghersalimi, and Z. Ghassemlooy, "Effect of transmitter and receiver parameters on the output signal to noise ratio in visible light communications," in 2017 Iranian Conference on Electrical Engineering (ICEE), 2017, pp. 2111-2116

[23] M. T. Niaz, F. Imdad, S. Kim, and H. S. Kim, "Deployment methods of visible light communication lights for energy efficient buildings," Opt. Eng., vol. 55, no. 10, p. 106113, Oct. 2016

[24] F. Li, K. Wu, W. Zou, and J. Chen, "Analysis of energy saving ability in dimming VLC systems using LEDs with optimized SAHP,” Opt. Commun., vol. 361, pp. 86-96, 2016

[25] F. Li, K. Wu, W. Zou, and J. Chen, "Optimization of LED's SAHPs to simultaneously enhance SNR uniformity and support dimming control for visible light communication," Opt. Commun., vol. 341, pp. 218-227, 2015

[26] L. A. Azizan, M. S. Ab-Rahman, M. R. Hassan, A. A. A. Bakar, and R. Nordin, "Optimization of signal-to-noise ratio for wireless light-emitting diode communication in modern lighting layouts," Opt. Eng., vol. 53, no. 4, p. 
45103, 2014

[27] T.-H. Do and M. Yoo, "Optimization for link quality and power consumption of visible light communication system,” Photonic Netw. Commun., vol. 27, no. 3, pp. 99-105, 2014

[28] H. Zheng, J. Chen, C. Yu, and M. Gurusamy, "Inverse design of LED arrangement for visible light communication systems," Opt. Commun., vol. 382, pp. 615-623, 2017

[29] A. Stavridis and H. Haas, "Performance evaluation of space modulation techniques in VLC systems," 2015 IEEE Int. Conf. Commun. Work. ICCW 2015, pp. 1356-1361, 2015

[30] A. M. Zaiton, H. R. A. Rahim, F. Jasman, and C. K. Yong, "Performance characterization of phase shift keying modulation techniques for indoor visible light communication system," in AIP Conference Proceedings, 2020, vol. 2203, no. January, p. 20023

[31] X. Gao, Z. Bai, P. Gong, and D. O. Wu, "Design and Performance Analysis of LED-Grouping Based Spatial Modulation in the Visible Light Communication System," IEEE Trans. Veh. Technol., vol. 9545, no. c, pp. 1-1, 2020

[32] L. Teixeira, F. Loose, J. P. Brum, C. H. Barriquello, V. A. Reguera, and M. A. D. Costa, "On the LED Illumination and Communication Design Space for Visible Light Communication," IEEE Trans. Ind. Appl., vol. 55, no. 3, pp. 3264-3273, 2019

[33] X. Xiao, H. Xiao, H. Liu, R. Wang, W. C. H. Choy, and K. Wang, "Modeling and Analysis for Modulation of Light-Conversion Materials in Visible Light Communication," IEEE Photonics J., vol. 11, no. 5, pp. 1-13, 2019.

[34] S. H. Lee, S.-Y. Jung, and J. K. Kwon, "Modulation and coding for dimmable visible light communication," IEEE Commun. Mag., vol. 53, no. 2, pp. 136-143, 2015

[35] V. Dixit and A. Kumar, "Performance analysis of non-line of sight visible light communication systems," Opt. Commun., vol. 459, no. September 2019, p. 125008, 2020

[36] M. S. M. Gismalla, M. F. L. Abdullah, M. Sami, N. S. M. Shah, B. Das, and A. A. Qasim, "Effect of Optical Attocells Deployment on the RMSD Spread in Indoor Visible Light Communication Systems," ICISCT 2020 2nd Int. Conf. Inf. Sci. Commun. Technol., 2020

[37] Z. Ghassemlooy, W. Popoola, and S. Rajbhandari, Optical wireless communications: system and channel modelling with Matlab®. CRC press, 2019

[38] M. S. M. Gismalla, M. F. L. Abdullah, W. A. Mabrouk, B. Das, and M. I. Niass, "Data rate and BER analysis for optical attocells configuration model in visible light communication," in 2019 International Conference on Information Science and Communication Technology, ICISCT 2019, 2019 